\title{
WILEY-VCH
}

DOI: $10.1002 /(($ please add manuscript number $))$

Article type: Communication

\section{A Lewis-Acid Monopolar Gate Dielectric for All-Inkjet-Printed Highly Bias-Stress Stable Organic Transistors}

Chen Jiang, Hanbin Ma, David Gregory Hasko, Xiaojun Guo, and Arokia Nathan*

C. Jiang, Dr. H. Ma, Dr. D. G. Hasko, Prof. A. Nathan

Department of Engineering, University of Cambridge, $9 \mathrm{JJ}$ Thomson Avenue, Cambridge

CB3 0FA, United Kingdom

E-mail: an299@cam.ac.uk

Prof. X. Guo

National Engineering Lab for TFT-LCD Materials and Technologies, Department of

Electronic Engineering, Shanghai Jiao Tong University, Shanghai 200240, People's Republic of China

Keywords: Lewis-acid monopolarity, bias-stress stability, inkjet printing, organic transistors

A Lewis-acid monopolar polymer with both hydrophobicity and lipophilicity is used as the gate dielectric for all-inkjet-printed organic thin-film transistors. The hydrophobicity of this polymer prevents water molecules from migrating and being trapped in the gate dielectric, while its lipophilicity allows good wetting by organic solvents for further deposition of other functional layers. These OTFTs demonstrate high bias-stress stability under both positive and negative bias in ambient air. This study unlocks the potential of all-inkjet-printed organic thin-film transistors for real-world low-cost large-area applications.

Organic thin-film transistors (OTFTs) have attracted considerable attention due to their lowcost printable processing, ${ }^{[1,2]}$ mechanical flexibility, ${ }^{[3]}$ and compatibility with arbitrary substrates. ${ }^{[4]}$ As a result, OTFTs are considered as the technology with the greatest potential for applications, such as sensors, ${ }^{[5]}$ radio frequency identification (RFID) tags, ${ }^{[6]}$ smart memories,${ }^{[7]}$ point-of-care diagnostic devices,${ }^{[8]}$ flexible display backplanes, ${ }^{[9]}$ and wearable systems. ${ }^{[10]}$ All-inkjet-printed (AIJP) OTFTs are in high demand, due to the advantages of drop-on-demand direct patterning, non-contact mode processing, reduced material wastage, 


\section{WILEY-VCH}

and high compatibility to large area manufacturing. ${ }^{[11,12]}$ However, despite these advantages, the bias-stress stability of AIJP OTFTs under realistic electrical operating conditions has not been addressed by any previous work, which is the prerequisite for their real-world applications.

The bias-stress instability of OTFTs can be attributed to intrinsic (e.g., structural and energetic disorder of the semiconductor) and extrinsic factors (e.g., oxidation, presence of moisture, and chemical impurities). ${ }^{[13,14]}$ By considering all these factors, the selection of the gate dielectric becomes essential, which could affect the device instability directly in both intrinsic and extrinsic ways. In general, in order to achieve high stability of OTFTs, the gate dielectric has to be low- $k$ and nonpolar, since a high- $k$ gate dielectric would cause semiconductor/dielectric interface dipole disorder, ${ }^{[15]}$ and a polar gate dielectric could induce water molecules to migrate or be trapped in the dielectric. ${ }^{[13]}$ Highly stable OTFTs have been achieved previously by using self-assembled monolayer (SAM) modification of gate dielectrics and/or low- $k$ fluoropolymer gate dielectrics. ${ }^{[16-18]}$ However, neither of these approaches is suitable for AIJP OTFTs. Inkjet printing technology requires good wetting properties for the depositing inks, so that polar materials with high surface energies are preferred. In all of the previous work involving AIJP OTFTs, ${ }^{[19-23]}$ poly(4-vinylphenol) (PVP) was used for the gate dielectric, since it is a polar polymer with high surface energy and is suitable for the following printing processes. However, PVP is particularly prone to moisture migration, leading to poor overall device stability. ${ }^{[24]}$

In this study, we have fabricated all-inkjet-printed highly bias-stress stable low-voltage OTFTs using a Lewis-acid monopolar polymer, namely polyvinyl cinnamate (PVCn), for the gate dielectric. Although PVCn has been used as a water repellent gate dielectric for OTFTs in other work, ${ }^{[25-27]}$ its hydrophobicity has not been fully explained. Here, we attribute the water repellency to the Lewis-acid monopolarity of PVCn. Due to its Lewis-acid monopolarity, PVCn not only exhibits a lower interaction energy with water than bipolar 


\section{WILEY-VCH}

materials, but also provides better wetting properties with most organic solvents than nonpolar materials. The fabricated AIJP OTFTs using PVCn as the gate dielectric demonstrate a low operating voltage $(<3 \mathrm{~V})$, a small subthreshold slope and a close-to-zero threshold voltage. Furthermore, the AIJP OTFTs using either PVCn or PVP gate dielectrics were bias-stressed under same operating conditions. The threshold voltage shift $\left(\Delta V_{\text {th }}\right)$ of PVCn-based OTFTs was less than $0.1 \mathrm{~V}$ after negative bias stressing for $2700 \mathrm{~s}$, while the $\Delta V_{\text {th }}$ of PVP-based OTFTs was more than $4 \mathrm{~V}$ after only 300 s. In addition, the PVCn-based OTFTs demonstrated faster recovery than the PVP-based OTFTs after exposure to light. Such good bias-stress stability of AIJP OTFTs is enabled by the hydrophobicity and the lipophilicity of PVCn.

The affinity of a liquid to a solid material greatly depends on their intermolecular interaction. In general, the intermolecular forces (including London dispersion, Keesom, and Debye) can be categorized into nonpolar and polar interactions. Water molecules, as bipolar dipoles with both Lewis acids and Lewis bases, tend to demonstrate polar interactions with neighboring molecules, while the organic solvents that are commonly used in inkjet printing normally exhibit nonpolar interactions (e.g. hexane, toluene) or Lewis-base-rich interactions (e.g. ethanol, ethylene glycol) with other molecules. ${ }^{[28]}$ In order to possess hydrophobicity and lipophilicity, a material should have a small Lewis-base interaction but a large Lewis-acid interaction. Therefore, the ideal water repellent gate dielectric for AIJP OTFTs should be a completely Lewis-acid monopolar material.

The polarity of PVCn and PVP were found by the electron density distributions around the surfaces of PVCn and PVP films, with illustrations of their electrostatic potential maps. Here, in order to investigate the properties of PVCn and PVP in the real-world application as gate dielectrics, the cross-linked (CL) structures of PVCn and PVP are used (Figure S1). The molecule geometries of CL-PVCn and CL-PVP were determined by molecular mechanics (extended MM2 force field), and their electrostatic potential maps were visualized using 


\section{WILEY-VCH}

extended Hückel molecular orbital calculation with WebMO. Figure 1a and 1b show the electrostatic potential maps of typical regions around the CL-PVCn surface and the CL-PVP surface, with red and blue representing electron-rich and electron-poor regions, respectively. The cross-linking sites are on the upper parts on the surfaces, due to the larger functional groups of these sites compared to the polyvinyl chains. As for CL-PVCn, it only contains electronegative oxygen atoms between the cross-linking sites and polyvinyl chains. This results in the electron-rich regions of PVCn being concealed underneath the surface of CLPVCn. As shown in the electrostatic potential tomography of CL-PVCn in Figure 1a, the top and bottom sections with phenyl rings and polyvinyl chains are electron-poor, while the fourth section dominated by the ester groups is electron-rich. This vertically changed electron density distribution is evidence of the Lewis-acid monopolar nature of CL-PVCn. As for CLPVP, both PVP itself and its cross-linking agent (i.e., poly(melamine-co-formaldehyde)) contain electronegative atoms (i.e., oxygen and nitrogen), which are located around the crosslinking sites. These electronegative atoms attract electrons from carbon atoms, resulting in the CL-PVP surface with both electron-rich and electron-poor regions, though the electron-rich (red) region is slightly larger. Therefore, CL-PVP behaves with bipolarity with both Lewis acids and Lewis bases.

In addition to the electrostatic potential mapping and tomography, the polarities of CL-PVCn and CL-PVP can be quantified by their nonpolar and polar components of surface energy, namely Lifshitz-van der Waals $\left(\gamma^{\mathrm{LW}}\right)$, Lewis-acid $\left(\gamma^{+}\right)$, and Lewis-base $\left(\gamma^{-}\right)$parameters. ${ }^{[29]}$ By using the sessile drop technique with three different polarity probe liquids (i.e., bipolar water, monopolar ethylene glycol (EG), and nonpolar hexane) as shown in Figure S2, the surface energy components for CL-PVCn and CL-PVP can be calculated by Equation S1, S2, S3 and S4. The contact angles and calculated results are shown in Table S1 and S2. Although these two dielectric materials exhibit similar nonpolar components, their polar components are different. The CL-PVCn shows a significant $\gamma^{+}\left(10.34 \mathrm{~mJ} \mathrm{~m}^{-2}\right)$ and a negligible $\gamma^{-}(0.01 \mathrm{~mJ}$ 


\section{WILEY-VCH}

$\mathrm{m}^{-2}$ ), which agrees with its Lewis-acid monopolarity; whereas the CL-PVP shows significant values for both $\gamma^{+}\left(8.85 \mathrm{~mJ} \mathrm{~m}^{-2}\right)$ and $\gamma^{-}\left(21.30 \mathrm{~mJ} \mathrm{~m}^{-2}\right)$. These differences in polar components lead to a lower total surface energy of the CL-PVCn compared to the CL-PVP, which are 16.46 and $43.42 \mathrm{~mJ} \mathrm{~m}^{-2}$, respectively. The low surface energy of CL-PVCn is essential for water repellency.

In order to evaluate the water repellency of CL-PVCn, its interaction energy $(\Delta E)$ with water was investigated by semi-empirical quantum chemistry calculations using the PM7 method with MOPAC. The interaction energy between CL-PVP and water was also studied for comparison. The geometries are defined in Figure $2 \mathrm{a}$ and $2 \mathrm{~b}$, respectively, with parameters of distance $(d)$ and rotation angle $(\alpha)$. For the CL-PVCn/water system, $d$ is the distance between the oxygen atom of the water molecule and the 4-position hydrogen atom of phenyl ring, and $\alpha$ is the angle between the phenyl plane and the water molecule plane. For CL-PVP/water system, $d$ is determined by the distance between the oxygen atom and the center of the melamine ring, and $\alpha$ is the angle between the normal direction of the melamine plane and the water molecule plane. The simulated interaction energy between CL-PVCn and water is shown in Figure 2c. As the water molecule approaches the CL-PVCn, the interaction energy decreases and then increases dramatically (Figure S3). This behavior is attributed to competition between an attractive dispersion force between molecules at long range and Pauli repulsion at short range. In addition, the interaction energy also changes with the rotation angle of the water molecule, which can be explained by the changes in the polar interaction during water molecule rotation. The interaction energy between CL-PVP and water shows similar approach and rotation behavior, as shown in Figure 2d. In general, CL-PVCn exhibited a smaller interaction energy with water (average value $-0.98 \mathrm{kCal} \mathrm{mol}^{-1}$ ) than $\mathrm{CL}-$ PVP (average value $-3.39 \mathrm{kCal} \mathrm{mol}^{-1}$ ), suggesting that CL-PVCn should be more water repellant than CL-PVP. 


\section{WILEY-VCH}

In addition to these differences, the two dielectric materials also show different behavior during water molecule rotation. Figure $2 \mathrm{e}$ shows the minimum water interaction energy with respect to rotation angle $\alpha$ of water molecule during approach. CL-PVCn has a high interaction energy with the $180^{\circ}$-rotated water molecule, but a low interaction energy with the $0^{\circ}$-rotated water molecule; these two conditions correspond to the interaction of the CL-PVCn with the Lewis base of water (i.e., oxygen atom) and with the Lewis acids of water (i.e. hydrogen atoms), respectively. This behavior can be attributed to the Lewis-acid monopolarity of CL-PVCn. Since CL-PVCn has few Lewis bases with a close-to-zero Lewisbase surface energy component, its interaction energy with the $0^{\circ}$-rotated water molecule stays approximately at zero. CL-PVP has a much higher interaction energy with the $0^{\circ}$-rotated water molecule than with the $180^{\circ}$-rotated water molecule, which although low is still considerable and is comparable to that for CL-PVCn, as shown in Figure 2e.

Figure $2 \mathrm{f}$ compares the calculated and experimental results showing the interaction between the two dielectric materials and water. In order to understand the effect of the polar interaction, a solely water Lewis-acid interaction and a solely water Lewis-base interaction with CLPVCn and CL-PVP were also investigated. For the solely water Lewis-acid interaction, the interaction energy is calculated when the water molecule is rotated to $180^{\circ}$, while the interfacial surface energy can be obtained by regarding the water molecules as fully polarized with Lewis acids facing the dielectric surfaces. The results for the solely water Lewis-base interaction were obtained similarly. As shown in Figure 2f, CL-PVCn and CL-PVP show similar interaction energies with solely water Lewis acids, with that for CL-PVCn being slightly higher. In contrast, the solely water Lewis bases demonstrate only weak interaction with CL-PVCn, but interact strongly with CL-PVP. Therefore, the overall interaction with water is weaker for CL-PVCn than for CL-PVP, which is consistent with the interfacial surface energies determined by experiment. 


\section{WILEY-VCH}

Though a low surface energy is important for water repellency, it is not sufficient to determine lipophilicity or lipophobicity. It is also noteworthy that the total surface energy for CL-PVCn $\left(16.46 \mathrm{~mJ} \mathrm{~m}^{-2}\right)$ is very close to that for CYTOP $\left(16.02 \mathrm{~mJ} \mathrm{~m}^{-2}\right){ }^{[30]}$ The surface energy consists of both polar and nonpolar contributions, and both CL-PVCn and CYTOP have a close-to-zero polar surface energy $\left(\gamma^{p}=2 \sqrt{\gamma^{+} \gamma^{-}}\right)$. In this case, without considering the Lewis-acid and Lewis-base interactions separately, CL-PVCn and CYTOP would be expected to give similar contact angles to water, EG, and hexane. However, the experimental results show that EG has better wetting properties on CL-PVCn than CYTOP, even though both repel water and are wetted by hexane (Table S1). For CYTOP, both the $\gamma^{+}$and $\gamma^{-}$are approximately zero, ${ }^{[30]}$ while PVCn, as a Lew-acid monopolar material, possesses a considerable $\gamma^{+}$, giving itself a higher Keesom interaction with other materials (particularly Lewis bases such as EG). According to Young's equation (Equation S1), a solid with low surface energy could provide good wetting properties to a liquid that has either a similarly low surface energy or a low solid-liquid interfacial surface energy. A low solid-liquid interfacial surface energy can be obtained by a combination of Lewis-acid solid and Lewis-base liquid, and vice vasa (Equation S3). Therefore, nonpolar solvents and Lewis-base-rich solvents can readily wet CL-PVCn, despite its low surface energy. Since the solvents used with inkjetprinted materials generally involve organics that are either nonpolar or Lewis-basedominated, ${ }^{[28]}$ CL-PVCn has good wetting properties for these solutions and so facilitates subsequent inkjet printing processes.

Based on the hydrophobicity and the lipophilicity of CL-PVCn, all-inkjet-printed (AIJP) highly bias-stress stable OTFTs have been demonstrated. The cross-section structure of the OTFTs and the chemical structure of the organic materials are shown in Figures $\mathbf{3 a}$ and $3 \mathrm{~b}$, respectively. A micrograph of a representative OTFT device with a channel width of $1200 \mu \mathrm{m}$ and a channel length of $20 \mu \mathrm{m}$ is shown in Figure 3c. Figures $3 \mathrm{~d}$ and $3 \mathrm{e}$ show typical transfer and output characteristics for these AIJP OTFTs with a mobility $(\mu)$ of $0.1 \mathrm{~cm}^{2} \mathrm{~V}^{-1} \mathrm{~s}^{-1}$, an 


\section{WILEY-VCH}

on/off ratio of $5.2 \times 10^{6}$, a threshold voltage $\left(V_{\text {th }}\right)$ of $0.05 \mathrm{~V}$, a subthreshold slope $(S S)$ of 116 $\mathrm{mV} / \mathrm{dec}$, and a semiconductor/dielectric interface trap density $\left(N_{\mathrm{Ss}}\right)$ of $7.78 \times 10^{10} \mathrm{eV}^{-1} \mathrm{~cm}^{-1}$. Such a small $N_{\text {SS }}$ was achieved by a blend of small molecule organic semiconductors with polymer binders, a smooth dielectric interface (Figure S4), and good wetting of semiconductor solvent on the dielectric (Figure S5). A small $N_{\text {SS }}$ is essential for a low operating voltage (i.e., 3V) of the fabricated OTFTs. ${ }^{[23,27,31,32]}$ Among the ever-reported AIJP TFTs, ${ }^{[19-23,33]}$ the fabricated OTFTs demonstrate the state-of-the-art performance, which is also comparable to the reported vacuum-processed and other solution-processed OTFTs (Table 1). ${ }^{[12,16,27,34,35]}$ In addition, these devices are also free from hysteresis in both transfer and output characteristics (Figure 3d and 3e), indicating a good stability of the structure (reduced semiconductor/dielectric interface traps and water molecule trapping during fabrication).

In order to further examine the stability of these OTFTs, we bias-stressed devices under realistic electrical operating conditions. Figure $3 \mathrm{f}$ shows the transfer characteristics of a typical AIJP PVCn-based OTFT device during a 45-min negative bias stress (i.e., $V_{\mathrm{GS}}=V_{\mathrm{DS}}=$ $-3 \mathrm{~V}$ ). During the bias stress, no significant shift (i.e., $-0.09 \mathrm{~V}$ ) in the transfer curves was observed, indicating a low level of traps in the device. In comparison, significant shifts in the transfer curves were observed in equivalent PVP-based OTFTs during 5-min and 45-min negative bias stress (Figure S7a and S7c). In addition, PVCn-based OTFTs also demonstrated a smaller transfer curve shift than found for PVP-based OTFTs during positive bias stress (i.e., $V_{\mathrm{GS}}=3 \mathrm{~V}, V_{\mathrm{DS}}=-3 \mathrm{~V}$ ), which is $0.13 \mathrm{~V}$ and $-0.42 \mathrm{~V}$, respectively, as shown in Figure S6 and S7. This level of operational stability under both negative and positive stress (i.e., $\left|V_{\mathrm{th}}\right|<0.13$ V) is comparable to the state-of-the-art bias-stress stable OTFTs and even vacuum-processed devices, which demonstrated $V_{\text {th }}$ shifts of several tenths volts (e.g., $0.3 \mathrm{~V}$ ) during $\sim 10^{3}$ second stress. $^{[12,27,36]}$ 


\section{WILEY-VCH}

A figure of merit to characterize the stability of OTFTs during bias stress is the threshold voltage shift $\left(\Delta V_{\text {th }}\right)$. Figure $3 g$ shows the $\Delta V_{\text {th }}$ for both PVCn-based and PVP-based OTFTs during negative and positive bias stresses. The $\Delta V_{\text {th }}$ over time $t$ can be expressed by a stretched-exponential function: ${ }^{[13]}$

$$
\Delta V_{\mathrm{th}}(t)=V_{0}\left(1-\exp \left(-\left(\frac{t}{\tau}\right)^{\beta}\right)\right)
$$

where $V_{0}$ is the maximum of $V_{\text {th }}$ shift (i.e., from the initial state to the infinitive state), $\tau$ is the time constant, and $\beta$ is the dispersion factor of an exponential distribution of trap states. By extracting the parameters (Table S3), we found that the PVCn-based OTFTs demonstrated a smaller $\left|V_{0}\right|$ and larger time constants than PVP-based OTFTs under both positive and negative bias-stress conditions, indicating the better stability of PVCn-based OTFTs. It is also worth noting that the directions of the $V_{\text {th }}$ shift were different between PVCn-based and PVPbased OTFTs. The PVCn-based OTFTs demonstrated a negative (positive) $\Delta V_{\text {th }}$ under negative (positive) bias stress, while PVP-based OTFTs, on the contrary, showed a positive (negative) $\Delta V_{\text {th }}$ under positive (negative) bias stress. These differences suggest that the stability of PVCn-based OTFTs is primarily influenced by intrinsic factors, whereas extrinsic factors (water molecules) are probably responsible for the instability of PVP-based OTFTs.

In order to demonstrate the stability of PVCn-based OTFTs in real-world applications, devices were bias stressed in an off state and their recovery from a period of light illumination was measured. A 365-nm UV LED was chosen as the illumination stress source, due to the strong absorbance of TIPS-pentacene/PS blend and the high transparency of CYTOP as seen in the UV-vis spectroscopy (Figure S8). As shown in Figure 3h, the PVCn-based OTFT demonstrated a stable photocurrent during illumination and fast recovery (with a time constant of $18 \mathrm{~s}$ ) to the baseline dark current after illumination. By contrast, the PVP-based OTFT demonstrated an increasing photocurrent during illumination and a slow recovery (with time constant of $327 \mathrm{~s}$ ) to a persistent photocurrent after illumination, as shown in Figure $3 \mathrm{i}$. 


\section{WILEY-VCH}

In conclusion, all-inkjet-printed (AIJP) bias-stress stable organic thin-film transistors (OTFTs) have been realized using a Lewis-acid monopolar polymer as gate dielectric, i.e. cross-linked polyvinyl cinnamate (CL-PVCn). This Lewis-acid monopolarity gives both hydrophobicity and lipophilicity. The hydrophobicity prevents water molecules from migrating and being trapped in the dielectric, and the lipophilicity enables the printability of other materials on top of the dielectric. The fabricated devices demonstrate high bias-stress stability under long-term realistic electrical operating conditions, unlocking the potential of AIJP OTFTs for real-world applications.

\section{Experimental Section}

Materials and ink formulation: The silver (Ag) ink (jet-600C) was supplied by Hisense Electronics, Kunshan, China, and the CYTOP (CTL-809M) and its solvent (CT-Solv. 180) were provided by Asahi Glass. All other chemicals were purchased from Sigma-Aldrich, including 6,13-bis(triisopropylsilylethynyl)-pentacene (TIPS-pentacene), polystyrene (PS), polyvinyl cinnamate (PVCn), poly(4-vinylphenol) (PVP), poly(melamine-co-formaldehyde) (PMF), and perfluorobenzenethiol (PFBT). The semiconductor ink was prepared by mixing TIPS-pentance $(15 \mathrm{mg})$ and PS $(5 \mathrm{mg})$ in toluene $(2 \mathrm{~mL})$. For dielectric inks, PVCn $(100 \mathrm{mg})$ was dissolved in anisole $(2 \mathrm{~mL})$, and PVP $(160 \mathrm{mg})$ and PMF $(80 \mathrm{mg})$ were dissolved in propylene glycol monomethyl ether acetate $(2 \mathrm{~mL})$. The CYTOP $(0.5 \mathrm{~g})$ was diluted with the CYTOP solvent (CT-Solv. 180, $1.5 \mathrm{~g}$ ), and then used as the ink for the encapsulation layer.

Device fabrication: All the ink-jet printing processes were conducted in ambient air with a Dimatix DMP-2831 inkjet printer, using $10 \mathrm{pL}$ droplet cartridges. The Ag ink was printed on a PVCn-coated $4 \mathrm{~cm} \times 4 \mathrm{~cm}$ glass substrate at a drop spacing of $50 \mu \mathrm{m}$ and then annealed at $150{ }^{\circ} \mathrm{C}$ for $15 \mathrm{~min}$ to form conductive gate electrodes. Then, the dielectric inks were printed at a drop spacing of $5 \mu \mathrm{m}$. The PVCn was crosslinked by being exposed to 254-nm UV light for 30 min, and the PVP was annealed at $150{ }^{\circ} \mathrm{C}$ for 2 hours. The source/drain electrodes were 


\section{WILEY-VCH}

formed by the same process used for the gate electrodes, and were then treated by immersing the samples into a mixed solution of PFBT $(1 \mu \mathrm{L})$ and ethanol $(1 \mathrm{~mL})$ for $3 \mathrm{~min}$ and rinsed with ethanol. The semiconductor was printed at a drop spacing of $5 \mu \mathrm{m}$, and followed by a 1 min baking at $100{ }^{\circ} \mathrm{C}$ to remove the excess of solvent. Finally, the devices were encapsulated with CYTOP before measurements.

Materials and device characterization: Fourier-transform infrared (FTIR) spectra of pristine PVCn and CL-PVCn were measured with a Perkin Elmer Frontier FTIR. UV-vis measurements of TIPS-pentacne/PS and CYTOP films on quartz substrates were made using a UniCam UV-vis spectrometer. The surface roughness of the CL-PVCn film was recorded with a Veeco EnviroScope atomic force microscope in a tapping mode. The electrical properties of the devices were characterized with a Keithley 4200 Semiconductor Characterization System.

\section{Supporting Information}

Supporting Information is available from the Wiley Online Library or from the author.

\section{Acknowledgements}

The authors acknowledge the support of the China Scholarship Council, Kangqiao Xingang Photonics Co. Ltd. (RG74597) and the British Council/DST UKIERI (RG72045).

Received: ((will be filled in by the editorial staff))

Revised: ((will be filled in by the editorial staff)) Published online: ((will be filled in by the editorial staff))

\section{References}

[1] R. A. Street, Adv. Mater. 2009, 21, 2007.

[2] H. Klauk, Chem. Soc. Rev. 2010, 39, 2643.

[3] M. Kaltenbrunner, T. Sekitani, J. Reeder, T. Yokota, K. Kuribara, T. Tokuhara, M. Drack, R. Schwödiauer, I. Graz, S. Bauer-Gogonea, S. Bauer, T. Someya, Nature 2013, 499, 458. 


\section{WILEY-VCH}

[4] H. Sirringhaus, Adv. Mater. 2014, 26, 1319.

[5] Y. Guo, G. Yu, Y. Liu, Adv. Mater. 2010, 22, 4427.

[6] V. Subramanian, J. M. J. Fréchet, P. C. Chang, S. Member, D. C. Huang, J. B. Lee, S. Member, S. E. Molesa, S. Member, A. R. Murphy, D. R. Redinger, S. Member, S. K. Volkman, Proc. IEEE 2005, 93, 1330.

[7] P. Heremans, G. H. Gelinck, R. Müller, K. J. Baeg, D. Y. Kim, Y. Y. Noh, Chem. Mater. 2011, 23, 341.

[8] P. Lin, F. Yan, Adv. Mater. 2012, 24, 34.

[9] I. Yagi, N. Hirai, Y. Miyamoto, M. Noda, A. Imaoka, N. Yoneya, K. Nomoto, J. Kasahara, A. Yumoto, T. Urabe, J. Soc. Inf. Disp. 2008, 16, 15.

[10] B. C.-K. Tee, A. Chortos, A. Berndt, A. K. Nguyen, A. Tom, A. McGuire, Z. C. Lin, K. Tien, W.-G. Bae, H. Wang, P. Mei, H.-H. Chou, B. Cui, K. Deisseroth, T. N. Ng, Z. Bao, Science 2015, 350, 313.

[11] M. Singh, H. M. Haverinen, P. Dhagat, G. E. Jabbour, Adv. Mater. 2010, 22, 673.

[12] K. Fukuda, Y. Takeda, Y. Yoshimura, R. Shiwaku, L. T. Tran, T. Sekine, M. Mizukami, D. Kumaki, S. Tokito, Nat. Commun. 2014, 5, 4147.

[13] H. Sirringhaus, Adv. Mater. 2009, 21, 3859.

[14] W. H. Lee, H. H. Choi, D. H. Kim, K. Cho, Adv. Mater. 2014, 26, 1660.

[15] J. Veres, S. D. Ogier, S. W. Leeming, D. C. Cupertino, S. M. Khaffaf, Adv. Funct. Mater. 2003, 13, 199.

[16] H. Klauk, U. Zschieschang, J. Pflaum, M. Halik, Nature 2007, 445, 745.

[17] W. L. Kalb, T. Mathis, S. Haas, A. F. Stassen, B. Batlogg, Appl. Phys. Lett. 2007, 90, 88.

[18] W. Zhang, Y. Han, X. Zhu, Z. Fei, Y. Feng, N. D. Treat, H. Faber, N. Stingelin, I. McCulloch, T. D. Anthopoulos, M. Heeney, Adv. Mater. 2016, 28, 3922.

[19] S. Chung, S. O. Kim, S. K. Kwon, C. Lee, Y. Hong, IEEE Electron Device Lett. 2011, $32,1134$.

[20] H. Y. Tseng, V. Subramanian, Org. Electron. 2011, 12, 249.

[21] E. Sowade, K. Y. Mitra, E. Ramon, C. Martinez-Domingo, F. Villani, F. Loffredo, H. L. Gomes, R. R. Baumann, Org. Electron. 2016, 30, 237.

[22] M. Medina-Sánchez, C. Martínez-Domingo, E. Ramon, A. Merkoçi, Adv. Funct. Mater. 2014, 24, 6291. 


\section{WILEY-VCH}

[23] L. Feng, C. Jiang, H. Ma, X. Guo, A. Nathan, Org. Electron. 2016, 38, 186.

[24] H. G. O. Sandberg, T. G. Bäcklund, R. Österbacka, H. Stubb, Adv. Mater. 2004, 16, 1112.

[25] J. Jang, S. H. Kim, S. Nam, D. S. Chung, C. Yang, W. M. Yun, C. E. Park, J. B. Koo, Appl. Phys. Lett. 2008, 92, 1.

[26] S. Lee, B. Koo, J. Shin, E. Lee, H. Park, H. Kim, Appl. Phys. Lett. 2006, 88, 2004.

[27] L. Feng, W. Tang, J. Zhao, R. Yang, W. Hu, Q. Li, R. Wang, X. Guo, Sci. Rep. 2016, $6,20671$.

[28] J. K. Fink, The Chemistry of Printing Inks and Their Electronics and Medical Applications, John Wiley \& Sons, Inc., Hoboken, NJ, USA, 2015.

[29] C. J. Van Oss, M. K. Chaudhury, R. J. Good, Chem. Rev. 1988, 88, 927.

[30] C. Jiang, H. Ma, D. G. Hasko, A. Nathan, Appl. Phys. Lett. 2016, 109, 211601.

[31] W. Tang, L. Feng, P. Yu, J. Zhao, X. Guo, Adv. Electron. Mater. 2016, 1500454.

[32] L. Feng, W. Tang, X. Xu, Q. Cui, X. Guo, IEEE Electron Device Lett. 2013, 34, 129.

[33] E. Sowade, E. Ramon, K. Y. Mitra, C. Martínez-Domingo, M. Pedró, J. Pallarès, F. Loffredo, F. Villani, H. L. Gomes, L. Terés, R. R. Baumann, Sci. Rep. 2016, 6, 33490.

[34] O. Acton, D. Hutchins, L. Árnadóttir, T. Weidner, N. Cernetic, G. G. Ting, T. W. Kim, D. G. Castner, H. Ma, A. K. Y. Jen, Adv. Mater. 2011, 23, 1899.

[35] A. Pierre, M. Sadeghi, M. M. Payne, A. Facchetti, J. E. Anthony, A. C. Arias, $A d v$. Mater. 2014, 26, 5722.

[36] T. Umeda, D. Kumaki, S. Tokito, Org. Electron. 2008, 9, 545. 
a
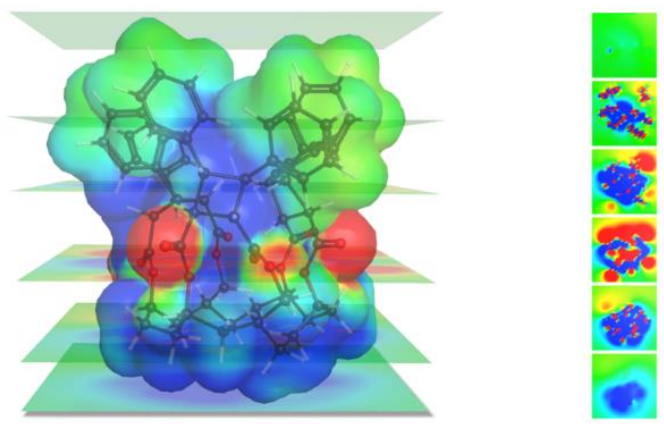

b
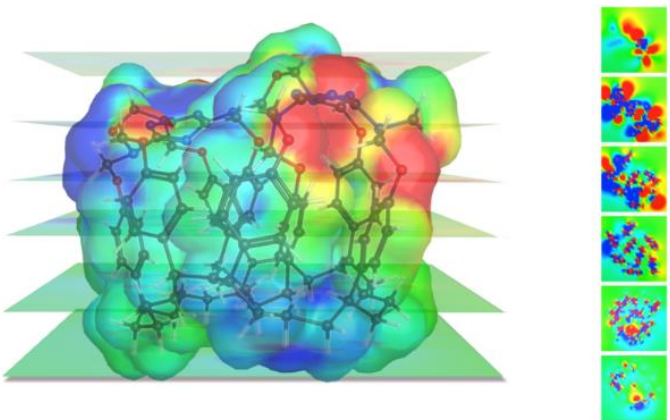

-0.04488 a.u.

0.04336 a.u.

Figure 1. 3D tomographic comparison of electron densities between CL-PVCn and CL-PVP. a, b) Electrostatic potential isosurfaces of typical cells of (a) CL-PVCn and (b) CL-PVP, with horizontal cuts representing the electron densities at discrete depths. The isosurfaces and tomographic sections are colored by red (electron-rich), green (neutral), and blue (electronpoor). Different atoms in the 3D molecular structures are colored gray (carbon), white (hydrogen), red (oxygen), and blue (nitrogen). 


\section{WILEY-VCH}
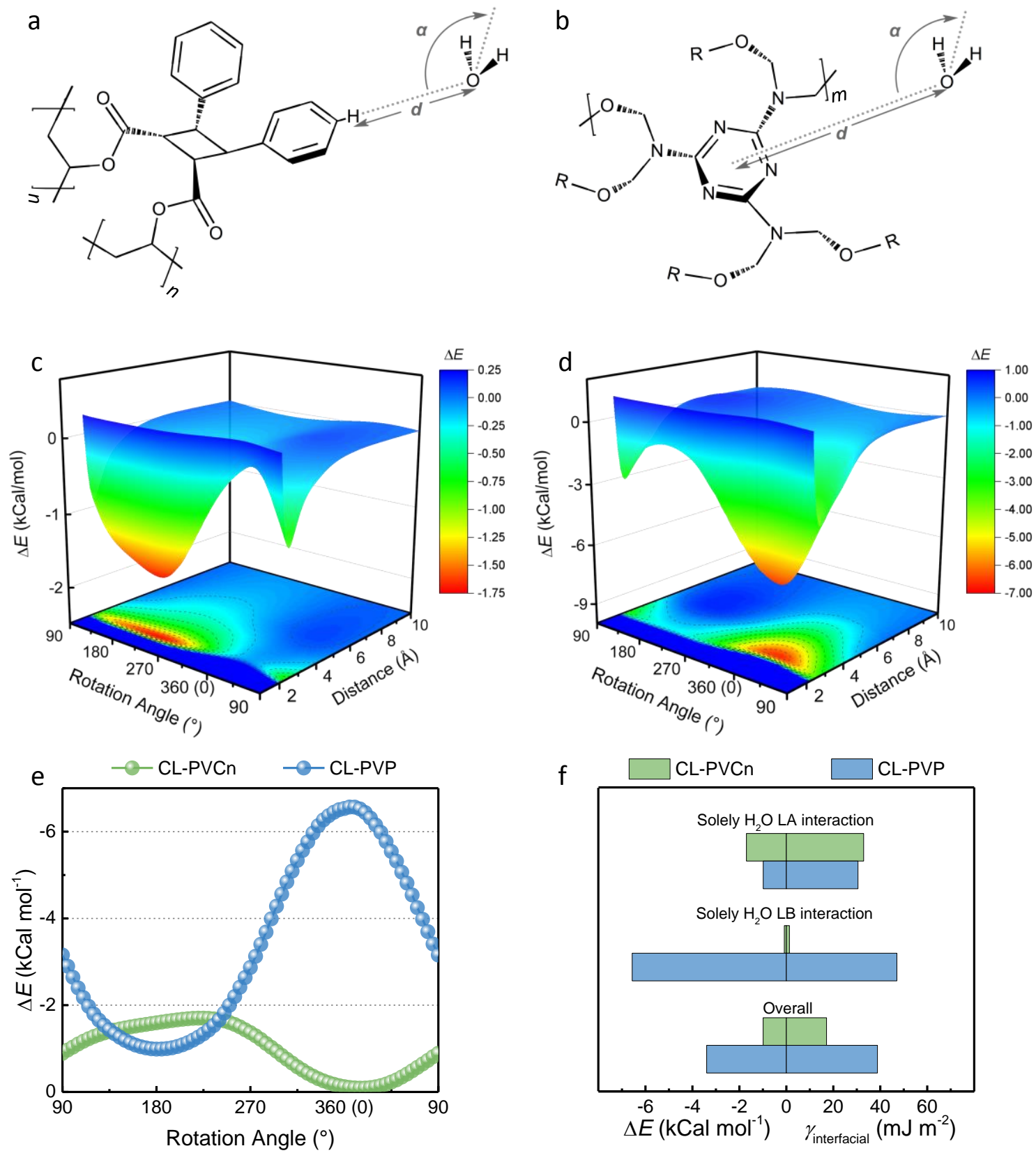

Figure 2. Interaction of water with CL-PVCn and CL-PVP. a,b) The geometries of (a) CL$\mathrm{PVCn} /$ water system and (b) CL-PVP/water system used in semi-empirical quantum chemistry calculations, with geometrical parameters of $d$ and $\alpha$. c,d) The interaction energy $(\Delta E)$ when a water molecule approaches (c) CL-PVCn and (d) CL-PVP at different rotation angles (from 0 to $360^{\circ}$ ). e) A comparison of minimum interaction energy of CL-PVCn/water and CL$\mathrm{PVP} /$ water at different rotation angles of a water molecule. f) The solely water Lewis-acid (LA) interaction, solely water Lewis-base (LB) interaction, and overall water interaction with CL-PVCn and CL-PVP, presented by the simulated interaction energy from semi-empirical quantum chemistry calculations and the interfacial surface energy from experimental data. The functional group of $\mathrm{R}$ in (b) is polyvinyl phenyl. 


\section{WILEY-VCH}

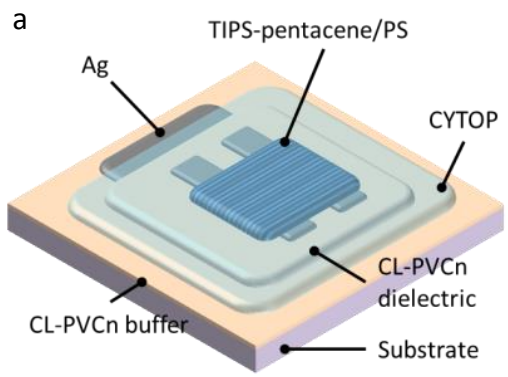

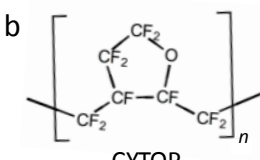

CYTOP
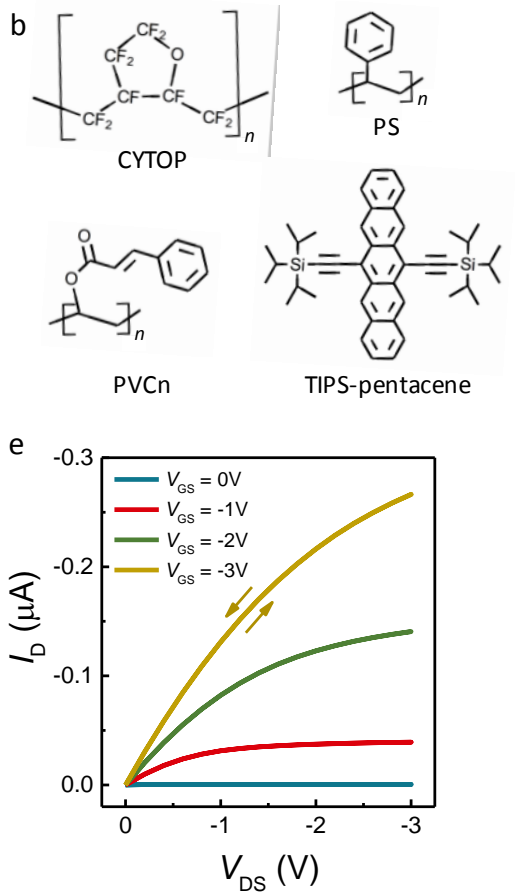

h

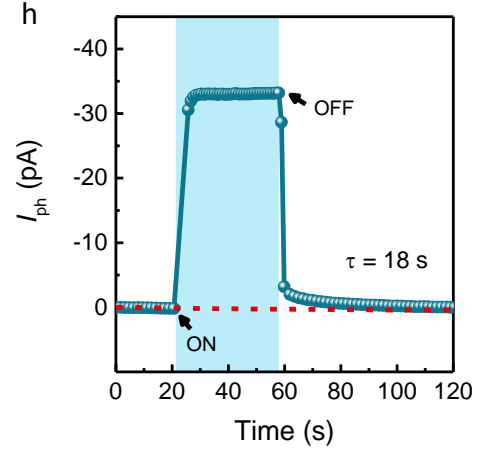

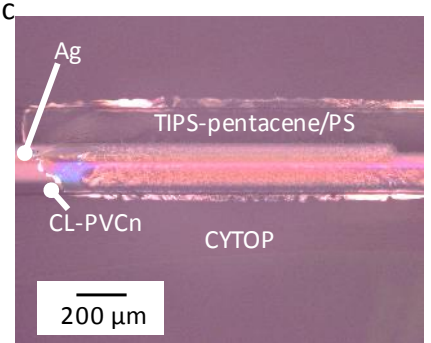

$2 \overline{00 \mu \mathrm{m}}$
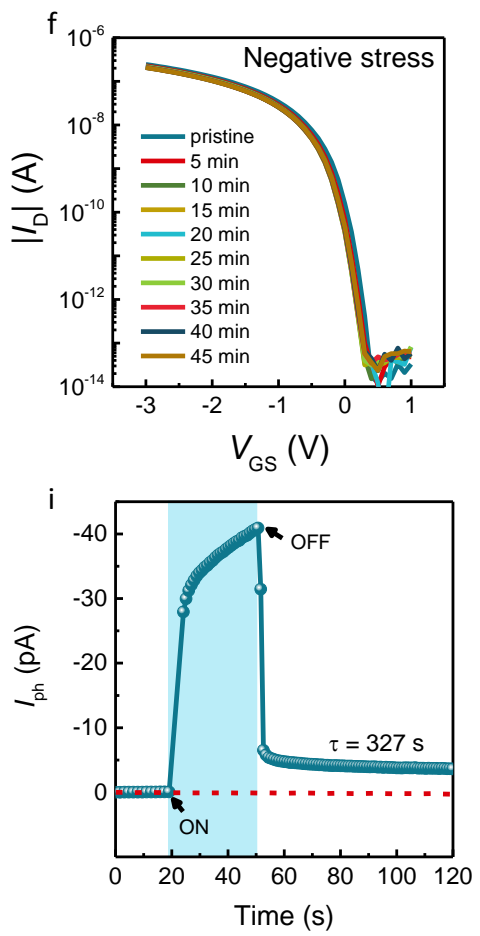

Figure 3. a) Schematic diagram of AIJP PVCn-based OTFTs and (b) the chemical structures. c) Polarized optical micrograph of a fabricated OTFT, with channel width of $1200 \mu \mathrm{m}$ and length of $20 \mu \mathrm{m}$. d,e) Measured (d) transfer and (e) output characteristics of a typical OTFT. f) Transfer curves for negative bias stressing $\left(V_{\mathrm{GS}}=V_{\mathrm{DS}}=-3 \mathrm{~V}\right)$ for the times indicated. $\mathrm{g}$ ) Threshold voltage shifts $\left(\Delta V_{\text {th }}\right)$ for PVCn-based and PVP-based OTFTs under negative and positive bias stressing. h,i) Photocurrent $\left(I_{\mathrm{ph}}\right)$ response for $(\mathrm{h})$ PVCn-based and (i) PVP-based OTFTs during and after a pulse of 365-nm UV illumination, with red dashed lines showing the corresponding baselines of dark current.

Table 1. Comparisons of mobility $(\mu)$, on/off ratio $\left(I_{\mathrm{on}} / I_{\mathrm{off}}\right)$, threshold voltage $\left(V_{\mathrm{th}}\right)$, subthreshold slope $(S S)$, and semiconductor/dielectric interface trap density $\left(N_{S S}\right)$ for this work and other reported OTFTs by different processes. 
WILEY-VCH

\begin{tabular}{ccccccc}
\hline Process & $\begin{array}{c}\mu \\
{\left[\mathrm{cm}^{2} \mathrm{~V}^{-1} \mathrm{~s}^{-1}\right]}\end{array}$ & $I_{\mathrm{on}} / I_{\text {off }}$ & $\begin{array}{c}V_{\text {th }} \\
{[\mathrm{V}]}\end{array}$ & $\begin{array}{c}S S \\
{[\mathrm{~V} / \mathrm{dec}]}\end{array}$ & $\begin{array}{c}N_{\mathrm{SS}} \\
{\left[\mathrm{eV}^{-1} \mathrm{~cm}^{-1}\right]}\end{array}$ & Ref. \\
\hline Vacuum-processed & 0.6 & $10^{7}$ & N/A & 0.100 & $3.0 \times 10^{12}$ & {$[16]$} \\
& 0.11 & $10^{5}$ & -0.7 & 0.100 & $1.91 \times 10^{12}$ & {$[34]$} \\
Solution-processed & 0.68 & $3.1 \times 10^{5}$ & -2.2 & 1.17 & $5.6 \times 10^{11}$ & {$[35]$} \\
& 0.95 & $4.8 \times 10^{6}$ & -0.68 & 0.486 & $3.9 \times 10^{11}$ & {$[12]$} \\
All-inkjet-printed & 0.6 & $10^{6}$ & -0.5 & 0.097 & $3.9 \times 10^{10}$ & {$[27]$} \\
& 0.02 & $10^{4}$ & -1.2 & 2.52 & $9.80 \times 10^{11}$ & {$[19]$} \\
& 0.01 & $1.4 \times 10^{3}$ & -1.5 & 4.28 & $1.50 \times 10^{13}$ & {$[20]$} \\
& 0.00082 & $5.5 \times 10^{1}$ & -14.0 & N/A & N/A & {$[33]$} \\
& 0.1 & $5.2 \times 10^{6}$ & 0.05 & 0.116 & $7.78 \times 10^{10}$ & Our work \\
\hline
\end{tabular}

N/A: Not available. 


\section{WILEY-VCH}

A Lewis-acid monopolar polymer with both hydrophobicity and lipophilicity is used as the gate dielectric for all-inkjet-printed highly bias-stress stable organic thin-film transistors. The hydrophobicity of this polymer prevents water molecules from migrating and being trapped in the gate dielectric, while its lipophilicity allows good wetting by organic solvents for further deposition of other functional layers.

Lewis-acid monopolarity, bias-stress stability, inkjet printing, organic transistors

C. Jiang, H. Ma, D. G. Hasko, X. Guo, A. Nathan*

A Lewis-Acid Monopolar Gate Dielectric for All-Inkjet-Printed Highly Bias-Stress Stable Organic Transistors

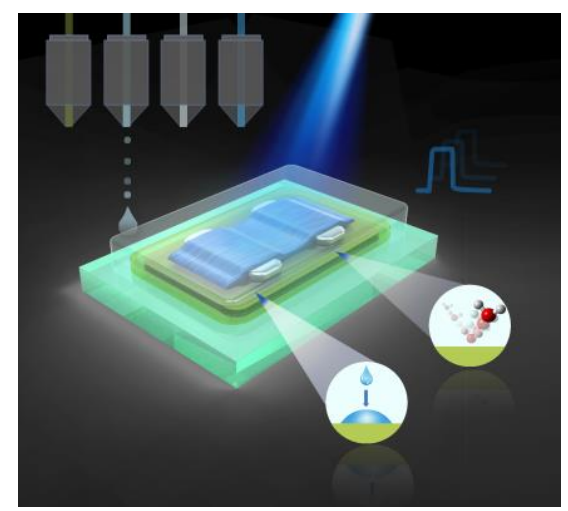

